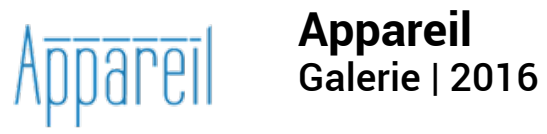

\title{
Architecture et musée : le passage chez Hubert Robert
}

Jean-Louis Déotte

\section{(2) OpenEdition}

Journals

Electronic version

URL: http://journals.openedition.org/appareil/2349

DOI: 10.4000/appareil.2349

ISSN: 2101-0714

\section{Publisher}

MSH Paris Nord

\section{Electronic reference}

Jean-Louis Déotte, «Architecture et musée : le passage chez Hubert Robert », Appareil [Online], Galerie, Online since 18 October 2016, connection on 30 July 2020. URL : http://journals.openedition.org/ appareil/2349; DOI : https://doi.org/10.4000/appareil.2349

This text was automatically generated on 30 July 2020 .

\section{(c) (i) (9)}

Appareil est mis à disposition selon les termes de la Licence Creative Commons Attribution - Pas d'Utilisation Commerciale - Pas de Modification 4.0 International. 


\title{
Architecture et musée : le passage chez Hubert Robert
}

\author{
Jean-Louis Déotte
}

\section{EDITOR'S NOTE}

Ce texte fut à l'origine un texte de catalogue pour l'exposition L'idée de musée organisée par le Musée de Valence en 1996. Que ses responsables d'alors soient remerciés, en particulier Mme Hélène Moulin, commissaire de l'exposition.

1 Hubert Robert a réuni trois compétences : architecte de jardins et de galeries, peintre d'antiquités, peintre de galeries et de jardins en ruine...

2 Comme architecte: des réalisations, beaucoup de projets, des édifices du passé qui auraient pu devenir autre chose que ce qu'ils sont devenus, des édifices à venir mais déjà ruinés. Et un peintre inquiétant, une temporalité trop sûre d'elle-même, trop causaliste : celle de l'historien d'art, souvent. Cette duplicité (double ou triple jeu de la temporalité) est plus que jamais nécessaire contre l'inquiétante tentation de remythologisation identitaire alors que tout échappe, et que les musées eux-mêmes sont appelés à devenir autre chose qu'eux-mêmes : banques de données informatisées ou centre d'animation culturelle.

3 Étant nécessairement post-hégélien comme tous ceux qui aiment les musées, considérant que ce sont les derniers lieux publics hospitaliers, ainsi qu'en convient de fait Thomas Bernhardt dans Les Maîtres anciens, nous ne pouvons que nous réjouir de cette nouvelle étape de la culture matérielle et de son historiographie que constitue l'informatisation des musées, la transformation des collections en banques de données immatérielles, voire l'existence d'archivage et de parcours de virtualités. Mais cette transformation, si elle concerne de fait le futur des médiathèques et des bibliothèques, n'affectera l'avenir du musée que par la bande, que par ce qui dans les œuvres est réductible à de l'information, disons pour rester imprécis, à ce qui n'est pas sa matière 
donc, ce qui est sa teneur chosale accessible à un savoir. En donnant ainsi une tout autre dimension au travail de l'historien d'art et donc au critique qui s'appuie sur lui.

4 Peut-être même la production artistique en sera-t-elle changée, puisque toute

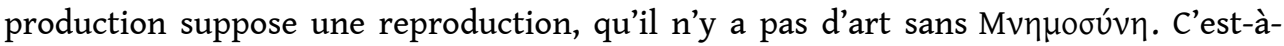
dire sans mémoire, très précisément parce que l'artiste ne travaille jamais dans un rapport immédiat au motif, naturel ou non, car il est toujours déjà enfermé dans la clôture de sa mémoire, donc toujours déjà aveugle (Derrida). Ou pour le dire autrement : qu'entre Cézanne et la Sainte Victoire, il y a toujours eu le Louvre.

5 L'effectivité du Musée qui nous intéressera, ce ne sera donc pas son devenirbibliothèque. Car, il y a une butée pour le musée, celle constituée par la matière des objets collectionnés et exposés.

6 Les points de fuite de plus en plus divergents, par exemple entre la bibliothèque et le musée du Centre G. Pompidou, leur quasi-divorce, surtout depuis la réforme de Gae Aulenti, sont à cet égard révélateurs, d'autant plus que les deux institutions étaient censées, dans le projet d'origine, se soumettre à une même loi, celle de l'actualité et de sa transparence communicationnelle. Le musée y avait été conçu comme une bibliothèque d'accès direct.

7 Or, l'actualité pour une bibliothèque, c'est l'actualité de l'événement politique et social, médiatisé, et l'actualité éditoriale, à la limite, celle des débats d'idées. C'est celle de l'information soumise de plus en plus à un principe de production médiatique de l'événement.

8 Cela étant, on n'ignore pas qu'il y a une part patrimoniale plus ou moins importante dans chaque bibliothèque et chez chaque bibliothécaire, mais cette dernière semble de plus en plus marginale, voire privée, comme l'est la passion du bibliophile.

9 L'actualité, même pour un musée d'art contemporain, ce devrait plutôt être celle du « maintenant ». Ce peut être celui du moment opportun - l'occasion - d'une exposition ou d'une étude, moment qui doit prendre la forme d'un sauvetage du passé. Dans ce sens, il n'est pas scandaleux que l'art contemporain ait aussi paradoxalement son musée, lequel est, on le sait, toujours musée d'histoire comme n'importe quel musée.

Si en effet il n'y a pas de paradoxe à ce que l'art ait comme réceptacle le Musée, même dans le cas des centres d'art contemporain, c'est que l'œuvre exposée, même la plus récente dans une chronologie linéaire, n'accédera au véritable maintenant qu'en entrant en correspondance avec un certain événement du passé, un Autrefois, une autre œuvre, une autre époque de l'art, les sauvant ainsi en exhibant leur parfaite contemporanéité avec elle.

11 C'est, me semble-t-il, l'enjeu des photographies contemporaines d'Elzéard, photographies d'un jardin, d'un musée, celui de Valence, de quasi-ruines, dans leurs rapports avec les dessins et peintures d'Hubert Robert. Dans ce sens, l'effectivité des musées, même d'art le plus contemporain, c'est bien toujours le passé. Le sauvetage du passé, à condition que l'on n'entende pas par là que tout doit revenir seulement parce qu'il est légitimé comme patrimoine. C'est dire que ce sauvetage ne peut se faire que sur un fond de discontinuité de l'histoire, qu'en en acceptant la ruine. Ce qui ébranle toute rétrospective naïve, toute familiarité avec le passé. Ce qui ne nous appartient plus, ce qui pour nous est passé dans le monde d'Hubert Robert - ce peintre qui met l'accent sur la ruinification du monde -, c'est précisément une familiarité avec les ruines, familiarité qui n'est plus la nôtre. Un véritable bonheur des ruines qui voit le 
petit peuple romain du $\mathrm{xvIII}^{\mathrm{e}}$ siècle faire son trou dans les vestiges colossaux de l'Empire. Au contraire, nous ne tolérons plus les ruines, nous n'en avons plus un culte, même sous la forme « moderne » que décrit Riegl dans son Culte moderne des monuments : nous ne sommes plus modernes, car nous ne prenons plus plaisir à la lente dégradation des édifices. C'est peut-être le symptôme d'une crispation identitaire métaphysico-politique post-moderne, si l'on juge à l'aune de ce qu'un Diderot attendait de la contemplation des peintures de ruines, d'Hubert Robert en particulier.

Chez Diderot, les ruines donnaient à penser, parce qu'elles présentaient les effets sur notre monde de l'accélération du temps, et, grâce au spectacle ruinant de notre monde, non pas le rappel d'un quelconque châtiment biblique, c'est-à-dire babylonien, mais une néantisation de ce monde-ci ouvrant au panorama spéculatif d'autres mondes possibles et parallèles. Par chance, laisse-t-il entendre dans la Lettre sur les aveugles, il y a d'autres mondes dans l'univers : nous ne les imaginons pas, trop fixés par l'apparence de pérennité de celui-ci, mais sachons voir dans l'aveugle ou le sourd et muet de naissance - ces monstres dans notre monde de voyants et d'entendants - les témoins isolés, ici, de mondes peuplés presque exclusivement là-bas d'aveugles et de sourds et muets et où, inversement, les voyants et les entendants terrestres seraient des handicapés majeurs. La ruine (de la vision, de l'ouïe, de la voix, de la raison, etc.) introduit à la pluralité des mondes, chaque infirmité se renverse en positivité. Il y a plus de vérité dans la ruine - qui introduit au devenir essentiel de toute chose - que dans le monument, intact, lequel, comme tout principe d'identité politique ou culturelle, nous berce de la rêverie du stable, de l'enracinement, dans la certitude d'être en propre ce qu'on est depuis toujours.

On voit bien que la simple propriété patrimoniale, par exemple des peintures d'Hubert Robert, ou le fait d'avoir ces œuvres transformées en stocks d'informations sous la main, détournent, silencieusement, d'un programme de pensée minoritaire. Au fond, n'importe quel chef-d'œuvre de nos musées est en train de se transformer en anecdote culturelle s'il n'est pas illuminé, au moins un instant, par le rayon laser d'une pièce contemporaine.

Ici, ce qui doit résister pour la pensée, ce qui fait matière, c'est le motif de la ruine.

De même qu'il y a une matière dans l'œuvre qui persiste en résistant, qui fait l'originalité du musée, la matière de cet événement passé qu'elle a été, donnant à penser, qui n'est donc pas réductible à une matière connue positivement, de même y at-il un certain passé vers lequel une pièce contemporaine peut jeter opportunément un pont, passé qui n'est pas patrimonial.

Ce qui se jouerait là à propos de la matière comme du passé, dans ce moment stratégique qu'est l'exposition de l'objet, c'est ce qui est dévolu comme tâche au Musée depuis l'inauguration du Louvre : réaliser, dans l'exposition, le destin de l'œuvre, en la libérant. Faire passer ce qui dans l'œuvre fut d'avènement, libérer le passage de l'événement. Ce qui consisterait, pour le collectionneur, non pas à se l'approprier en la faisant sienne, mais au contraire, à l'habiter.

17 C'est bien une affaire d'exposition, de scénographie, un savoir-faire d'espacement, une question d'espace au moins dans les premiers temps, telle qu'entre l'œuvre du Maintenant et celle du passé jaillisse une sorte d'arc dialectique, une image dialectique selon Benjamin, une tension sensible à tous, visible et invisible comme toute différence, une césure, une césure de l'espace et du temps. L'interprétation spatiale d'une œuvre 
est un art de la césure temporelle, du passage temporel. Comme architecture, le Musée est donc bien déjà, dans tous les sens du terme, un passage. Le passage du Musée.

Passage qu'il faudrait dérouler depuis sa fin, depuis les œuvres les plus récentes, parce que la marche à reculons (comme dans l'exposition, il y a quelques années, de la collection Ménil à Paris), est beaucoup plus pertinente : l'Autrefois ne peut être connu que du point de vue du plus contemporain. Tout simplement parce que les artistes contemporains se voient rivés à une seule tâche: citer à comparaître des réalités passées. Comme des prophètes qui tournent leurs yeux vers le passé.

Le musée est, en France, mais certainement aussi à l'étranger, ce qui dans le genre " architecture publique » a été le plus enrichi. À un point tel qu'on peut se demander s'il est possible de penser le mode de vie urbain postmoderne en dehors de lui.

Si Benjamin a pu penser la préhistoire de la modernité en analysant ce type particulier d'architecture urbaine du premier quart du XIX siècle que fut le passage urbain, il nous reviendrait aujourd'hui, entrant dans une nouvelle étape de la fantasmagorie moderne (la post-modernité), de considérer de manière essentielle un autre type de passage urbain, où la rue s'est aussi comme invaginée, où les façades sont comme rentrées, où les fenêtres n'ouvrent que sur d'autres salles qui sont comme autant de mondes intérieurs, mais où ce ne sont plus des marchandises qui s'exposent au flâneur, mais ces pièces que l'on peut appeler des suspens : des objets de musée.

21 Des pièces dont nous avons hérité du passé ou de l'exogène, comme aussi bien des productions de l'art contemporain. Pièces, et non œuvres parfaitement achevées, qui ont en commun d'être des fragments de ces mondes. Et on a pu spontanément les considérer comme des expressions de ces mondes et non comme leurs reflets (Benjamin sur le collectionneur et historien Fuchs). Telle œuvre de tel artiste exprimerait la totalité de sa production, appelée aussi son œuvre, comme cette œuvre d'une vie exprimerait une époque de l'art, comme cette époque serait comme un monde, etc. Or, devant ces expressions, le visiteur passe.

Car le musée est bien une galerie exigeant le passage le plus banal : en effet, si devant une œuvre de culte, dans un temple, c'est-à-dire devant une œuvre destinale (même dans le cas du culte de l'art pour l'art), on effectue une génuflexion, devant un suspens, un fragment, dans un musée, on passe.

On sait que si, pour l'essentiel, les passages de Paris qui intéressèrent tant Benjamin furent construits dans le premier tiers du XIX ${ }^{\mathrm{e}}$ siècle, leurs prototypes picturaux furent, eux, le fait d'Hubert Robert. Et l'on pourrait, à la suite de la recherche d'André Corboz', établir une systématique des architectures en "tunnel » qui permettrait d'articuler structuralement des éléments tirés de La découverte de Laocoon (1775), de La Statue de Ménandre dans une colonnade antique voûtée (1773), Intérieur d'un passage (1760), La Grotte de Pausilippe (1769), Vue de l'entrée de la grotte de Pausilippe près de Naples (1781), Lavandières dans une galerie antique (1760), Troupeau sous une voûte en ruine, Temple antique (1787), L'ermite, Galerie ruinée, Caprice avec des statues du Capitole, Arc de Triomphe, Les Fontaines (1787), Ruines avec lavandières, Galerie avec lavandières, Variation sur la Scala Régis du Vatican, L'Escalier des lavandières, Canal dans un parc français, Scène au bord d'un canal, Le canal, La promenade de Madame Geoffrin à l'Abbaye Saint-Antoine, Intérieur d'une galerie antique en ruines, Une galerie de musée consacrée à l'art de la Rome antique ou le Musée (1780), Projet pour l'aménagement de la Grande Galerie du Louvre (1796), jusqu'à la très fameuse Grande Galerie du Louvre en ruine ou la Vue d'une salle du Musée des Monuments 
français (1800). Mais la clef d'un tel ensemble ne nous semble pas être une «poétique des axes rapides $»^{2}$ qui relève trop d'une problématique "ponts et chaussées » de la communication. On peut soupçonner, au contraire, que le grand nombre des versions, ruinées ou non, du «tunnel », de la "galerie », du «passage », est plutôt l'expression d'une contradiction logique, qu'il s'agirait de cerner.

À titre d'éléments, car là n'est pas le but de notre réflexion, on peut suggérer que le thème du "tunnel » élève à la dimension architecturale et métaphysique le motif de l' absorbement que Michael Fried ${ }^{3}$ place au cœur de la critique picturale de Diderot, s'agissant précisément de peintures comme celles d'Hubert.

La Ville-monde ne serait rien d'autre que cette multiplicité des musées qui l'exprime et ainsi chaque musée, du haut de sa singularité discernable, exprimerait tel point de vue sur la Ville, suivant telle perspective. Celle de sa collection aujourd'hui exposée. Mais en même temps, la Ville n'étant que ce réseau, chaque musée exprimerait un certain état historique du réseau des collections. Les musées devraient alors nécessairement s'entr'exprimer, l'état des collections exprimées étant relatif à toutes les autres expositions. Cette co-appartenance des musées en tant qu'ils s'entr'expriment, que s'entr'exprimant, ils expriment le monde, suppose bien une sorte de site commun qui pour les monades est, selon Leibniz, reprenant un terme emprunté à la perspective, le géométral. Dieu, écrit-il, est le géométral des monades. Qu'en sera-t-il des musées? Sont-ils d'essence théologique? Difficile d'être ici intégralement leibnizien.

Il y a là la question du site commun, de l'universel, que suppose n'importe quel échange entre les hommes, question qui déborde donc en apparence largement la question du Musée.

Les économistes libéraux résolvent le problème du site commun aux activités humaines en invoquant le marché ; les disciples de Marx, à l'auto-production de l'homme grâce au travail, ceux de Merleau-Ponty, la chair du visible, ceux d'Hannah Arendt, la certitude d'être du même monde en même temps du fait de voir la même chose, ceux d'Habermas, la visée commune d'un même consensus de vérité, ceux de Jean-Luc Nancy, l'entr'exposition des singularités, les démocrates, les droits de l'homme, etc.

Les uns et les autres se réfèrent en fait, selon des pentes très différentes, au présupposé d'une expérience commune qui nous serait encore accessible, une terre natale.

Or, sans cultiver le catastrophisme ou la nostalgie, il faut bien admettre que nous sommes héritiers d'un gigantesque champ de bataille, que nous sommes pour cela hommes de la ruine, ruinés-ruinant. Entre l'expérience irreprésentable du front de la Première Guerre mondiale et la Shoah, il nous est loisible de «choisir » notre terrain natal. Disons que c'est à cette aune, celle de l'expérience de l'effondrement de l'expérience, pour parler comme Françoise Proust commentant Benjamin, que nous pouvons jauger et invalider les définitions citées précédemment du site commun.

Condamnés alors à la caverne muséale, contemplant non pas les ombres d'une présence inaccessible, mais héritiers sans destin de ruines qui ne nous permettent pas d'avoir, au premier abord, une relation vivante avec le passé, donc de renouer avec la tradition, de nous relier légitimement au passé et en cela donc, sans religion.

31 Dès lors, les objets de musée ne peuvent être ni des reliques ni des symboles. Ils ne représentent pas, dans le visible, l'invisible. Ce ne sont pas des sémiophores. Mais toujours objet de deuil dans un jeu de deuil (Trauerspiele) dont Benjamin a montré qu'il 
était à l'origine du drame baroque allemand. C'est-à-dire des allégories. Et comme il s'agit d'objets, d'allégories matérielles.

S'installant dans la cavité muséale, c'est-à-dire dans notre époque d'inclusion passionnée, toute d'immanence, sans transcendance et sans destination, sans étoiles la nuit puisque notre regard est arrêté par la coupole lumineuse de la ville, ne s'agit-il pas alors de redonner un écrin, un réceptacle, un ornement (un jardin) à nos existences? Ne s'agit-il pas alors, déjà pour les pièces fragmentaires que nous avons recueillies, de leur redonner une chance d'être des événements, de leur permettre au moins de rejouer le deuil de l'événement qu'elles furent en leur temps? Ne faut-il pas alors creuser la montagne de Salzbourg comme le projeta l'architecte Hans Hollein pour son musée, parce que l'architecture de musée ne saurait être qu'intérieure, la seule capable peut-être de transmettre ce qui peut l'être encore? Cela qui nous donnera non pas le site commun d'une expérience collective, mais comme un héritage de restes énigmatiques à déchiffrer ensemble? Il n'est donc pas étonnant que, pour une bonne part de l'art contemporain, la caverne muséale s'invagine toujours plus avant, que le grand ornement de l'art abrite des pièces comme les Igloos de Mario Merz, Plitght de Beuys, Le Jardin d'hiver de Dubuffet, les Cabanes éclatées de Buren ou même la Salle blanche de Broothaers ${ }^{4}$. Comme si la rumeur spontanée des pièces ne pouvait être perçue que dans de tels réceptacles. Du fait de leur discernabilité, comme au Musée de Vérone de Scarpa. De leur entr'expression, comme au musée Picasso de Simounet.

\section{BIBLIOGRAPHY}

Corboz, André, Peinture militante et architecture révolutionnaire. À propos du tunnel chez Hubert Robert, ETH, Editions Dirkhauser, Dôle, 1970.

Fried, Michael, La place du spectateur. Esthétique et origines de la peinture moderne, Paris, Gallimard, 1990, trad. fr. Claire Brunet.

\section{NOTES}

1. André Corboz, Peinture militante et architecture révolutionnaire. À propos du tunnel chez Hubert Robert, ETH., éditions Dirkhäuser, Dôle, 1970.

2. Ibid., p. 40.

3. Michael Fried, La Place du spectateur. Esthétique et origines de la peinture moderne [1980], trad. de l'anglais (États-Unis) par Claire Brunet, Paris, Gallimard, 1990

4. Au Musée National d'Art Moderne. Centre Georges Pompidou. 


\section{ABSTRACTS}

Alors que notre époque déteste les ruines, à moins de les faire entrer dans le patrimoine, une exposition récente du Louvre (été 2016) consacrée à Hubert Robert, met en exergue cet objet temporel par essence. Mais comme en outre, Hubert Robert a eu l'audace d'imaginer la Grande Galerie en ruines, alors qu'elle n'existait comme lieu d'exposition qu'à l'état de projet, on peut affirmer qu'il a fourni l'allégorie picturale d'une temporalité qui, ignorant le présent de l'actualité, celui de la causalité, ne considère que le passé et l'avenir. La temporalité muséale par excellence.

INDEX

Mots-clés: ruine - archive - musée - maintenan- passage urbain 